\title{
FACTORS INFLUENCING THE CONNECTION OF ACRYLIC TEETH WITH DENTURE PLATE
}

\author{
Zbigniew Raszewski
}

R \& D, SpofaDental, Jičín, Czech Republic

\begin{abstract}
INTRODUCTION: Patients who use removable dentures often contact dentists about restoration of a separation or fracture of their acrylic teeth. This causes great discomfort for the patient as well as huge labor and time-consuming procedures for dentists and dental technicians.

Овјестіves: The purpose of this study was to evaluate the adhesion between self-curing acrylic material and teeth in distillate water and denture disinfection agent.

MATERIAL AND METHODS: Five types of self-cure acrylic resin samples' groups were prepared for the study, and were combined with acrylic teeth. First group included teeth samples made without surface modification, second group consisted of teeth samples' surfaces activated with acetone, while in third group, retention holes were made. In fourth group of samples, their surfaces were increased by retention holes and washed twice with monomer, whereas in fifth group of teeth, cervical layers were grinded with a bur. One plate from each group was stored in distilled water (W) and another in Corega Tabs (CT) solution for 30 days at $37^{\circ} \mathrm{C}$. Connection between acrylic resin-acrylic tooth was assessed using tensile strength instrument.

RESULTS: The highest bonding force was achieved between Villacryl SP and Mifam teeth in the group with retention holes made and surface washed twice with monomer (W: $149.86 \pm 5.42 \mathrm{~N}$; CT: $146.38 \pm 4.39 \mathrm{~N}$ ), cohesive type. The lowest adhesion values were observed for tooth surfaces with no changed surface (W: $15.15 \pm 3.44 \mathrm{~N}$; CT: 13.49 $\pm 3.80 \mathrm{~N}$ ), and with samples washed with acetone (W: $15.8 \pm 3.22 \mathrm{~N} ; \mathrm{CT}: 12.0 \pm 1.0 \mathrm{~N}$ ). In these cases, the connection had adhesive character.

ConCLUsions: A proper connection between acrylic teeth and surface of denture base is possible to achieve when both chemical and mechanical methods surface are combined, thus increased by retention elements.
\end{abstract}

KEY wORDS: self-curing acrylic resin, acrylic teeth, adhesion, denture cleaning agent.

J Stoma 2021; 74, 4: 249-253

DOI: https://doi.org/10.5114/jos.2021.111289

\section{INTRODUCTION}

Patients who use removable dentures often contact dentists about restoration of a separation or fracture of their acrylic teeth. This causes great discomfort for the patient as well as huge labor and time-consuming procedures for dentists and dental technicians $[1,2]$. Process of separating the teeth from acrylic surface can be divided into two ways, including preparation of a tooth surface during polymerization of new denture and during using of prosthesis. Depending on the type of polymerization, acrylic resins may have different adhesion to acrylic teeth. There is a large number of publications in the literature, but the conclusions are equivocal [1-5]. Some authors report that polymerized materials present a bond with acrylic, others argue that

\section{JOURNAL OF STOMATOLOGY CZASOPISMO STOMATOLOGICZNE}

ADDRESS FOR CORRESPONDENCE: Zbigniew Raszewski,

R \& D, SpofaDental, Markova 238, 506-01, Jičín, Czech Republic,

e-mail: Zbigniew.Raszewski@kavokerr.com

ReCEIVED: 07.04.2021 • ACCePted: 22.06.2021 • Published: 30.11 .2021 
the teeth are separated from acrylic in a cohesive manner $[3,4,6]$. Such differences may be influenced by polymerization condition of self-cure (SC) materials and preparation of the tooth surface. During polymerization, SC materials are subjected to low pressure from 0.2 to $0.6 \mathrm{MPa}$ (pressure unite) and temperature from 40 to $60^{\circ} \mathrm{C}$, for a period of $15-30$ minutes [6]. In contrast, heat cure materials are polymerized in more demanding conditions, and polymerization process can be done under a pressure of 1,500-2,000 kg and longer time from 60 minutes to 7 hours at a high temperature $[1,4]$.

Numerous authors emphasize the importance of proper preparation of tooth surfaces before polymerization. For this purpose, they suggest to increasing the surface of teeth through mechanical retentions (sandblasting or laser treatment), or chemical activation (treatment with monomer, acetone, or different bonding agent) $[1,5,6-9]$.

During use, patients who take care of their daily hygiene of their restorations, clean dentures with various types of denture cleaners, which may be indifferent to acrylic surface. According to the literature, it is possible to find information on the effect of denture disinfection agents on mechanical properties and the color of acrylic materials. Various authors have observed a decrease in fracture resistance, an increase in surface roughness, and a change in color after long-term exposure to agents based on hypochlorite or active oxygen [10-13]. The authors of the present study, however, did not manage to find information on how disinfectants for dentures can affect the connection between teeth and denture plate.

In order to broaden the knowledge about the correct connection of teeth with denture base, made with pouring acrylic, it was decided to examine the connection be-

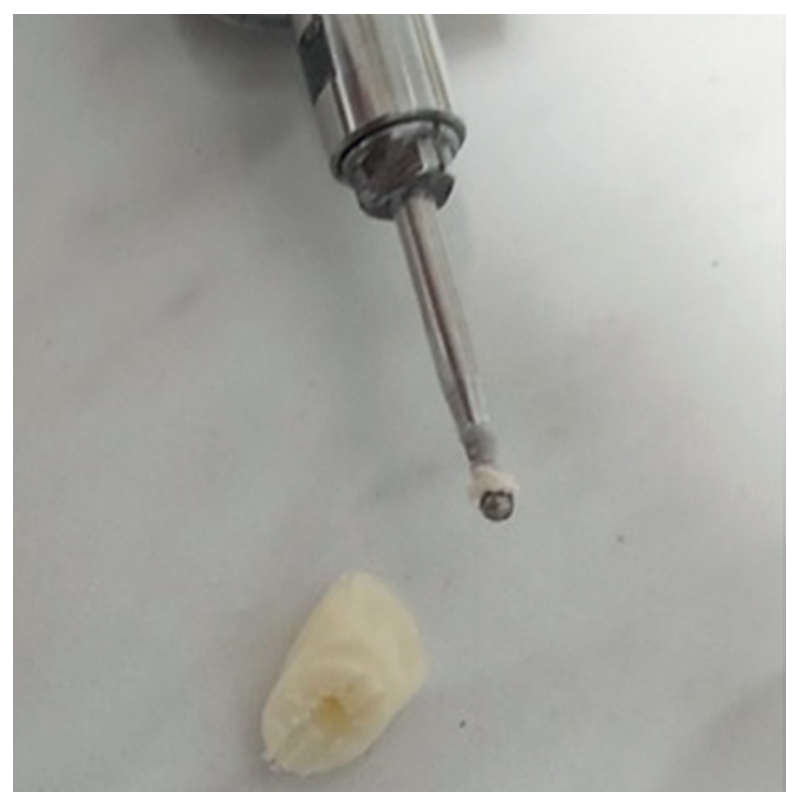

FIGURE 1. Tooth surface prepared with bur with visible retention hole tween Villacryl SP and acrylic teeth by expanding their surfaces with various methods, and storing the samples in distillate water and denture disinfection agent.

The hypothesis of this work was that the method of preparing the surfaces of the teeth has a major impact on the proper connection between acrylic resin and acrylic tooth. The second thesis to be proved was that denture disinfectants may affect the connection of acrylic teeth with denture plate, as they affect the acrylic surface itself, which has been proven in previous studies.

\section{MATERIAL AND METHODS}

As an example of acrylic material for the pouring technique, resin Villacryl SPV4 (batch Z091457, expiry date October, 2024, Zhermapol; Warsaw Poland) was used. The material, according to the manufacturer's instruction, is hardened under a pressure of 2.5 bar in water, at a temperature of $55^{\circ} \mathrm{C}$ for 30 minutes. As a representative material for acrylic teeth (AT), incisors Mifam Super Lux, N-5 color (Spofa Dental; Jicin, Czech Republic) were applied. In order to investigate the connection of the teeth with acrylic, the methodology described in the ISO 20795-1:2013 standard was applied [14]. Fifty teeth were used for the study, which were divided into 5 groups. First group, as a reference, was used without further preparation; in second group of acrylic teeth, surface was grounded with a bur cutter H1S Komet (Brasseler; Seevetal, Germany). Third group samples were equipped with retention holes (Figure 1), whereas fourth group of AT had retention holes, and their surfaces were washed twice with a monomer (180 s) after wax boiling phase. In fifth group of acrylic teeth, samples were twice chemically treated with acetone (Sigma Aldrich; Poland).

Villacryl SP material was mixed in a proportion of $10 \mathrm{~g}$ of powder and $6.7 \mathrm{~g}$ of liquid, using a Denver 300 laboratory balance (Denver, Colorado, USA) calibrated until June, 2021. The powder and monomer before mixing, had a temperature of $23^{\circ} \mathrm{C}$, which was checked with a certified thermometer (Tech MM; California, USA). After 2 minutes (time was checked by JVD System stopwatch; Nove Mesto, Czech Republic) from the moment of mixing of the acrylic material, it was poured into a plaster mold made of plaster Stodent III (Zhermapol; Warsaw, Poland).

Previously, after wax boiling out and washing with boiling water, the gypsum mold was insulated with a gypsum acrylic separator (Izodent, SpofaDental; Czech Republic) after wax boiling out.

After 4 minutes, when a thin harder layer appeared on the acrylic surface, the whole was placed in a polymerization chamber Ivomat (Icolar Vivadent; Liechtenstein). Polymerization process took place in water temperature of $65^{\circ} \mathrm{C}$, for 30 minutes, and pressure of $2.5 \mathrm{bar}$.

For each of the groups (1-5), two acrylic plates were made. The first ones were stored in distilled water for 
30 days at $37^{\circ} \mathrm{C}$ at laboratory drier Biological Thermostat BT-50 (Brno, Czech Republic). The second plate was stored in the solution of Corega Tabs (Corega) denture cleaner. A tablet was dissolved in a $200 \mathrm{ml}$ of distilled water. Every 3 days, water was removed and a new tablet was diluted in new portion of water. Acrylic plates with denture cleaner samples (6-10) were stored for 30 days at $37^{\circ} \mathrm{C}$ in the same equipment as groups $1-5$. This process was supposed to simulate the long-lasting impact of disinfection tablets on acrylic surfaces [13]. In total, 10 samples with 50 acrylic teeth were prepared for the experiment.

After this period of time, the samples were placed in a manual tear machine Famed 4 (Vaclav Lapka; Brno, Czech Republic), and subjected for a tear test. The force increased at a rate of $1 \mathrm{~mm} / \mathrm{min}$. The test ended when the connection between the tooth and the acrylic plate was broken. The results of teeth separation from prosthesis' plate were read from the dial in Newtons $(\mathrm{N})$.

Statistical analysis was performed using two-way ANOVA test, with post-hoc Tukey HSD (honestly significant difference) test calculator using unmodified tooth sample as a reference. $P<0.05$ coefficient was accepted as the confidence level. Free software from a website was applied for statistical processing (https://astatsa. com/OneWay_Anova_with_TukeyHSD/).

\section{RESULTS}

Results from the tests are presented in Table 1. Additionally, photographs of the detached acrylic teeth from the denture plate were taken, as showed in Figures 3 and 4.

The adhesion of acrylic teeth with an unmodified surface or washed with acetone showed the lowest level of adhesion to the surface of the denture plate, stored both in distilled water and in solution for denture disinfection. The strength of the connection ranged from $15.15 \pm 3.44 \mathrm{~N}$ for distillate water and $13.49 \pm 3.80 \mathrm{~N}$ for Corega Tabs (Figure 4). The strongest connections were observed for surfaces of teeth with retention holes and washed with monomer. The obtained connection was cohesive in nature (Figure 3 ). In this case, the values of adhesion were $149.86 \pm 5.42 \mathrm{~N}$ for distilled water and $146.38 \pm 4.39 \mathrm{~N}$ for Corega Tabs.

Statistical analysis showed statistical differences $(p<0.01)$ within one group, depending on the method

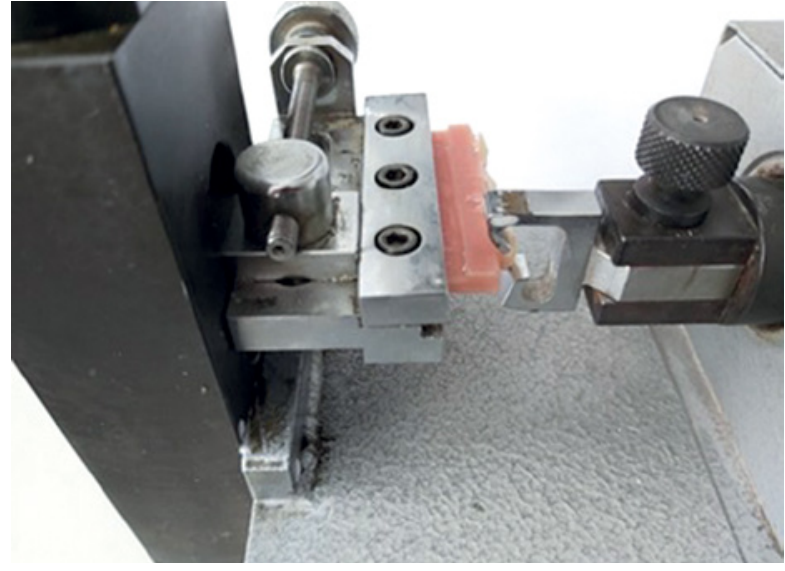

FIGURE 2. Testing procedure

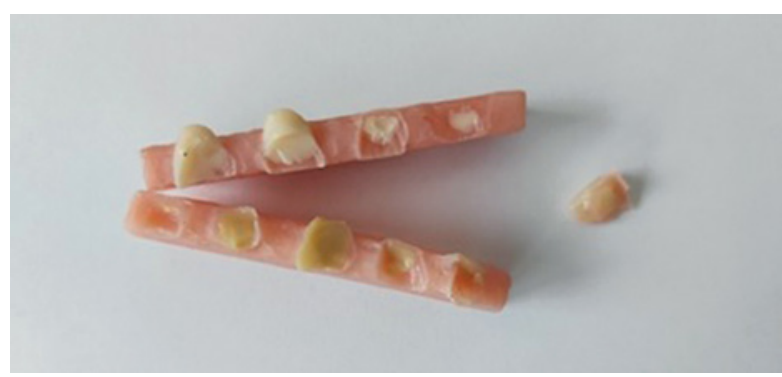

FIGURE 3. Cohesive connection between acrylic teeth (mechanical retention and monomer treatment)

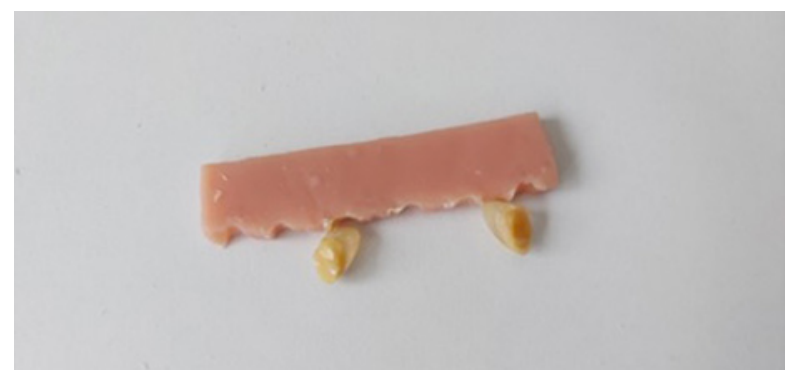

FIGURE 4. Adhesive debonding that occurred in samples with acetone and no treatment group

of tooth surface preparation. However, they were not significant between two investigated groups, i.e., distillate water and Corega Tabs. Teeth adhesion in the group

TABLE 1. Tests results of acrylic teeth adhesion to self-curing resin in comparison to no treatment samples. Force value in $\mathrm{N}$

\begin{tabular}{|c|c|c|c|c|c|}
\hline & Non treatment & Surface grind with bur & Retention holes & $\begin{array}{l}\text { Retention holes } \\
\text { with } 2 \text { times monomer }\end{array}$ & $2 \times$ acetone \\
\hline 30 days distillate water & $15.15 \pm 3.44$ & $58.64 \pm 4.28$ & $115.83 \pm 3.44$ & $149.86 \pm 5.42$ & $15.8 \pm 3.22$ \\
\hline$p$-value* & & $<0.01$ & $<0.01$ & $<0.01$ & \\
\hline 30 days Corega Tabs & $13.49 \pm 3.80$ & $56.65 \pm 4.18$ & $107.95 \pm 7.84$ & $146.38 \pm 4.39$ & $12.0 \pm 1.0$ \\
\hline$p$-value ${ }^{*}$ & & $<0.01$ & $<0.01$ & $<0.01$ & \\
\hline
\end{tabular}


with samples immersed in Corega Tabs solution were lower by nearly $5 \%$ than in the comparative group, but not statistically significant.

\section{DISCUSSION}

The first part of hypothesis presented at the beginning of this study was confirmed, with acrylic presenting different adhesions depending on the method of surface preparation, which is consistent with results of other authors $[4,5]$. The second part of the question posed as to whether the disinfectants may interfere with the connection between the tooth and the denture plate has not been confirmed. In a study, Muhsin et al. noticed that teeth with retention pores presented with over $42.50 \mathrm{MPa}$ adhesion, which was more than 2 times higher than the connection between teeth with an unmodified surface of $27.46 \mathrm{MPa}$. According to the same authors, it is very important to wash the teeth surfaces with the use of monomer [8]. The longer the contact time with the monomer, the better the adhesion. This phenomenon was explained by the fact that methyl methacrylate can dissolve the surfaces of acrylic teeth. In this study, the authors applied two-time monomer until it completely evaporated. The total contact time with monomer was measured with a calibrated stopwatch, established for 180 seconds. In Vallittu et al. [7], contact of the monomer with the tooth surface produced formation of numerous pits up to $2 \mu \mathrm{m}$ in diameter. In order to achieve that, a specific time of 180-240 seconds of direct exposure to the monomer on the tooth surfaces was required [8].

Other authors in their tests used solvents designed to dissolve polymethyl methacrylate - acetone, ethyl acetate, or chloroform. Their results indicated that there was an improvement in the bond between the teeth and the denture plate $[15,16]$. In the current study, the increase of the surface was small, it was most likely due to the fact that acetone is a substance that evaporates easily, and the contact time was too short to effectively dissolve the surface layer of teeth.

Kumar et al. investigated the connection of teeth to self-cure and hot curing. Better adhesion was noticed with the first type of material, when the surface was washed with a monomer. In case of thermally polymerized materials, the authors did not notice an increase in the connection between the teeth and the denture plate after using a monomer. This result explains that self-cure polymers contain a plasticizer that absorbs the monomer more easily [16].

Azhagarasan et al. observed a very large improvement in the bonding of teeth to acrylic resin in extension of mechanical surface of teeth by using a cutter or sandblasting [17]. This is consistent with the results of the current study, where the surface of the teeth was extended with cutter along with making retention holes.
In their research, Choudhry et al. showed a connection of acrylic teeth with acrylic prosthesis, with correctly boiling out the wax from the tooth surfaces. During the study, they used Raman's spectroscopy for detection of wax remains on teeth surfaces. Their conclusions demonstrated that even on the surface of teeth grind with a cutter there may be residues of undissolved wax. Moreover, if water used to dissolve the wax has a temperature of $80-90^{\circ} \mathrm{C}$, the residual wax content is even higher. Therefore, it is recommended to use detergents in order to better clean the surfaces of teeth [18]. During the current study, the wax was removed by boiling it in hot water for 10 minutes (calibrated stopwatch), washing the tooth and plaster surfaces with detergent, and rinsing twice with $95^{\circ} \mathrm{C}$ hot water (calibrated thermometer).

Authors who conducted a research on denture disinfection agents noted affected surfaces of acrylic materials [10-13]. Additionally, sodium hypochlorite solutions have a more destructive effect $[12,13]$. Agents based on active oxygen (Corega Tabs) do not show such a large impact on mechanical resistance of acrylics [13]. Therefore, during these tests, no effect of this agent was observed at the junction of the denture plate and acrylic teeth. Also in this case, the tooth groups 1 and 5 (without mechanical modification of surface) showed a connection of the adhesive type, and the tooth was separated from the denture plate.

Over a prolonged period of in vivo use, the strength of the connection between the teeth and the denture plate may be influenced by other factors, including temperature changes, food chewing, use of brushes, and abrasive toothpastes [1]. Some of them can be assessed in laboratory conditions (thermocycling), others require in vivo examination [18-22].

\section{CONCLUSIONS}

The combination of mechanical adhesion using retention elements and chemical interaction by partial dissolution of acrylic teeth surfaces with a monomer, allows for a proper connection of the acrylic teeth to the acrylic plate, made of a self-cure material. Denture disinfectants do not adversely affect the adhesion of the teeth to the denture plate.

\section{CONFLICT OF INTEREST}

The authors declare no potential conflicts of interest with respect to the research, authorship, and/or publication of this article.

\section{Reference}

1. Sanjayagouda B, Naveen N. Bonding acrylic teeth to acrylic resin
denture bases: a review. Gerodontology $2006 ; 23: 131-139$. 
2. Mahadevan V, Krishnan M, Krishnan CS, et al. Influence of surface modifications of acrylic resin teeth on shear bond strength with denture base resin-an invitro study. J Clin Diagn Res 2015; 9: ZC16-ZC21. DOI: 10.7860/JCDR/2015/13877.6445.

3. Zidan S, Silikas N, Haider J, Alhotan A, Jahantigh J, Yates J. Assessing tensile bond strength between denture teeth and nano-zirconia impregnated PMMA denture base. Int J Nanomedicine 2020; 15: 9611-9625.

4. Al Zaher ZA, Almaskin DF, Qaw MS, et al. Chemo-mechanical approach to improve repair bond strength of denture teeth. Int J Dent 2020; 2020: 8870361. DOI: https://doi.org/10.1155/2020/8870361

5. Han SY, Moon YH, Lee J. Shear bond strength between CAD/ CAM denture base resin and denture artificial teeth when bonded with resin cement. J Adv Prosthodont 2020; 12: 251-258.

6. Al-Musawi R. The effect of different curing time on the impact strength of cold and hot-cure acrylic resin denture base material. Med J Babylon 2014; 11: 188-194.

7. Vallittu PK, Ruyter IE, Nat R. The swelling phenomenon of acrylic resin polymer teeth at the interface with denture base polymers. J Prosthet Dent 1997; 78: 194-199.

8. Muhsin SA. Bond strength of repaired acrylic denture teeth using visible light cure composite resin. Open Dent J 2017; 11: 57-64.

9. Malkoç MA, Demir N, Ögreten AT, Ozturk AN, Kiliç HS. Effect of new laser type on shear bond strength of acrylic teeth to denture base. J Res Dent 2015; 3: 26-30.

10. Ragher M, Vinayakumar G, Patil S, et al. Variations in flexural strength of heat-polymerized acrylic resin after the usage of denture cleansers. J Contemp Dent Pract 2016; 17: 322-326.

11. Peracini A, Davi LR, de Queiroz Ribeiro N, et al. Effect of denture cleansers on physical properties of heat-polymerized acrylic resin. J Prosthodont Res 2010; 54: 78-83.

12. Carvalho C, Vanderlei A, Salazar Marocho S. Effect of disinfectant solutions on a denture base acrylic resin. Acta Odontol Latinoam 2012; 25: 255-260.

13. Pereira CJ, Genari B, Leitune VCB. Effect of immersion in various disinfectant solutions on the properties of a heat-cured acrylic resin. Rev Gaúch Odontol 2019; 67: e20190052. DOI: https://doi. org/10.1590/1981-86372019000523090.

14. ISO 20795-1:2013, Dentistry - Base polymers - Part 1: Denture base polymers. Available at: https://www.iso.org/standard/62277.html.

15. Gowda S, Kumar L, Sehgal K, et al. Comparative evaluation of effect of pre-processing surface treatment of acrylic teeth on bonding with denture base resins-an in vitro study. Int J Oral Care Res 2018; 6: 13-18.

16. Kumar PA, Iniyan $\mathrm{K}$, Balasubramaniam R, Viswanathan $\mathrm{M}, \mathrm{Hi}$ nes PAJ, Monnica V. The effect of surface treatments on the shear bond strength of acrylic resin denture base with different repair acrylic resin: an in vitro study. J Pharm Bioallied Sci 2019; 11 (Suppl 2): S380-S384

17. Mahadevan V, Krishnan M, Krishnan CT, Azhagarasan NS, Sampathkumar J, Ramasubramanian H. Influence of surface modifications of acrylic resin teeth on shear bond strength with denture base resin - an invitro study. J Clin Diagn Res 2015; 9: ZC16-ZC21.

18. Choudhry Z, Malik S, Khan S, Ahmed MA. Bonding of acrylic resin teeth with denture base resin. Pakistan Oral \& Dental Journal 2015; 35: 757-761.

19. Marra J, Freitas de Souza R, Barros Barbosa D, Pero AC, Compagnoni MA. Evaluation of the bond strength of denture base resins to acrylic resin teeth: effect of thermocycling. J Prosthodont 2009; 18: 438-443.

20. Adeyemi AA, Lyons MF, Cameron DA. The acrylic tooth-denture base bond: effect of mechanical preparation and surface treatment. Eur J Prosthodont Restor Dent 2007; 15: 108-114.

21. AlZaher ZA, Almaskin DF, Qaw MS, et al. Chemo-mechanical approach to improve repair bond strength of denture teeth. Int J Dent 2020; 2020: 8870361. DOI: https://doi.org/10.1155/2020/8870361.

22. Jain G, Palekar U, Awinashe V. The effect of different chemical surface of denture teeth on shear bond strength. J Clin Diagn Res 2014; 8: ZC15-ZC18. 